Check for updates

Cite this: RSC Adv., 2019, 9, 35872

Received 16th September 2019 Accepted 30th October 2019

DOI: $10.1039 / c 9 r a 07437 f$

rsc.li/rsc-advances

\section{Aggregation-induced emission enhancement (AIEE)-active boron-difluoride dyes with reversible mechanochromic fluorescence $\uparrow$}

\author{
Xiaojing Yan, ${ }^{a}$ Pengcheng Zhu, ${ }^{a}$ Zhiguo Zhou, (D) ${ }^{\mathrm{b}}$ Hong Yang, ${ }^{\mathrm{b}}$ Haichuang Lan ${ }^{\mathrm{a}}$ \\ and Shuzhang Xiao (D) *a
}

A novel class of solid-emissive boron-difluoride derivatives, using phenanthrenequinone hydrazone as ligands, were designed and efficiently synthesized. These dyes exhibit weak fluorescence in dilute solutions, but much higher fluorescence efficiency in aggregate states with a large stokes shift (over 70 $\mathrm{nm}$ ) due to the their aggregation-induced emission enhancement (AIEE) characteristics. According to their photophysical properties and X-ray single crystal structure analysis, the AIEE was ascribed to the $\mathrm{H}(\mathrm{J})$-aggregate formation aided by multiple intermolecular interactions to restrict intramolecular motion in the solid state. Moreover, their solid emissions could be reversibly tuned between "on" and "off" by mechanical grinding and recrystallization, due to the stacking model transition between $H(J)$ aggregation with loose molecular packing and J-aggregation with intense intermolecular interactions.

\section{Introduction}

Fluorescent dyes have been receiving continuous attention for their potential applications in advanced science and technology. Although large number of highly fluorescent chromophores have been developed during the past decades, borondifluoride dyes are still of great interest due to their numerous advantages, such as high fluorescence intensity, excellent chemical- and photochemical-stability, long excitedstate lifetime and narrow emission spectra. ${ }^{1}$ However, typical boron-difluoride dyes (BODIPY, namely 4-bora-4,4-difluoropyrromethene) exhibit highly planar chemical structures, which would facilitate the formation of $\pi-\pi$ stacking and lead to aggregate-induced fluorescence quenching. Moreover, the high symmetry character of BODIPYs makes the potential energy surfaces of the ground and excited state less distinct. ${ }^{2-4}$ Therefore, BODIPY dyes possess small stokes shift and are usually non-emissive in the solid state.

For the purpose of practical applications in photoelectric devices, it's quite important to maintain their emissions in the solid state and increase their Stokes shifts. Up to now, there have been a few techniques to construct solid-emissive boron-

${ }^{a}$ College of Biological and Pharmaceutical Sciences, China Three Gorges University, Yichang 443002, Hubei, P. R. China. E-mail: shuzhangxiao@ctgu.edu.cn; Fax: +86 7176397478

${ }^{b}$ The Education Ministry Key Lab of Resource Chemistry, Shanghai Normal University, Shanghai 200234, China

$\dagger$ Electronic supplementary information (ESI) available: Additional photophysical data, figures and table, crystal structure analysis. CCDC 1946768. For ESI and crystallographic data in CIF or other electronic format see DOI: 10.1039/c9ra07437f difluoride complexes, such as introducing bulky groups around the $\mathrm{BF}_{2}$ chelate to alleviate the intermolecular $\pi-\pi$ stacking by steric effect and designing intermolecular noncovalent bonding to promote non-parallel packing. ${ }^{5}$ During the recent years, we have adopted the second technique to develop a series of boron-difluoride dyes based on the ligand of 2-(2'-pyridyl) imidazole derivatives, and found that these borondifluoride dyes (BOPIM) exhibit quite large stokes shift (over $100 \mathrm{~nm}$ ) and high fluorescence intensity in the solid state. ${ }^{6-11} \mathrm{It}$ seems that low-symmetrization of the structures is quite effective to cause more energetically distinguished ground and excited states to enlarge the Stokes shift. ${ }^{12}$ However, the synthesis of BOPIM dyes is tedious because of the low synthetic yields. On the other hand, the chemical stability of BOPIM dyes needs to be enhanced because they tend to decompose under basic conditions.

During the recent years, limited number of solid-emissive boron-difluoride dyes with aggregation-induced emission enhancement (AIEE) have been reported. AIEE substances were first developed by Tang et al. in 2001, which emits weak fluorescence when dissolved, but strong luminescence in the aggregate state.$^{13}$ Fluorescent dyes with AIEE properties have many advantages for practical application in the fields of analytical detection, fluorescence imaging and luminescent devices. For AIEE-active boron-difluoride dyes, non-pyrrolebased ligands with asymmetrical structures were normally adopted, such as $\beta$-diketone or $N$-substituted $\beta$-diketone derivatives, ${ }^{14-18} \beta$-iminoenolate derivatives ${ }^{19-27}$ and others. ${ }^{28-35}$ All these dyes bear rotatable bulky aromatic groups, which promotes the non-irradiative decay in dilute solutions. But in the solid state, the rotation was restricted, therefore, they 
emit low or no fluorescence in dilute solutions but intense fluorescence in the solid state. Although a few examples of AIEE complexes ascribed to $\mathrm{J}$ aggregation, ${ }^{36,37}$ excimer formation $^{38-40}$ have been reported, there have been limited reports on AIEE boron-difluoride dyes based on $\mathrm{J}(\mathrm{H})$ aggregate which are formed between chromophores with tight chromophore spacing and strong coupling, ${ }^{41}$ and it is still highly significant to explore new AIEE dyes based on boron-difluoride chromophore for developing new functional luminescent materials. On the other hand, the aggregation and deaggregation might be manipulated by external stimuli (heat, pressure and etc.) to provide environment-sensitive materials. Herein, we designed and synthesized two novel boron-fluoride derivatives with AIEE properties and investigated their mechanochromic properties.

\section{Experimental}

\subsection{Materials and methods}

All starting materials were obtained from commercial supplies and used as received. ${ }^{1} \mathrm{H}$ NMR spectrum was recorded on Bruker 400 NMR instruments, using $\mathrm{CDCl}_{3}$ as solvent. PXRD diagrams were tested on a D8 ADVANCE (Bruker). UV-vis and fluorescent measurements were carried out on Shimadzu UV2600 and Hitachi F-4600, respectively. The fluorescence quantum yields in solutions are calculated using rhodamine $\mathrm{B}$ as a reference, and the fluorescence quantum yields of the solid powders were measured from a Hamamatsu Absolute PL Quantum Yield Spectrometer C11347. Size distribution were measured on a Malvern Zetasizer (Nano ZS90). Single crystal of PHPO-BF 2 suitable for X-ray diffraction measurements was obtained by slow evaporation from a mixture of dichloromethane and petroleum ether. X-ray diffraction data were collected on a BRUKER D8 QUEST diffractometer. And the CCDC number is 1946768. $\dagger$ The initial powder samples for mechanochromism study are obtained by vacuum evaporation of column eluents, and ground samples were obtained by grinding in a mortar manually.

Geometry optimization were performed by density functional theory (DFT) at B3LYP with the $6-31+\mathrm{G}(\mathrm{d}, \mathrm{p})$ basis sets. ${ }^{42}$ The search for potential crystal structures was performed by Accelrys Polymorph Predictor module of the Materials Studio software, using the simulated annealing algorithm of Karfunkel and Gdanitz. ${ }^{43}$ The molecular cluster with intense intermolecular $\pi-\pi$ interactions in single crystal was chosen as input and calculated at the M06-2X/6-31+G(d,p) level with dispersion correction (empirical dispersion $=\mathrm{GD} 3) .{ }^{44}$ Then intermolecular interactions were analyzed by Multiwfn and visualized by VMD. ${ }^{45}$ The intermolecular interactions were shown as green clouds.

\subsection{Synthesis}

Precursors in Scheme 1 were prepared according to literature procedure ${ }^{46}$ The precursors $(6.7 \mathrm{mmol})$ and triethylamine $(48.3 \mathrm{mmol}, 6.7 \mathrm{~mL})$ were dissolved in anhydrous dichloromethane $(30.0 \mathrm{~mL})$ and stirred at ice bath for $10 \mathrm{~min}$ under an atmosphere of argon. Then boron trifluoride etherate $(73.8 \mathrm{mmol}, 9.1 \mathrm{~mL})$ was added, and the resultant mixture was stirred at room temperature overnight. After the reaction was quenched with water and then extracted by dichloromethane, the organic phase was dried over anhydrous sodium sulfate and then evaporated under vacuum. The obtained crude product was purified by recrystallization from a mixture of dichloromethane and petroleum ether to afford red solids (yield: $95 \%$ for $\mathbf{P H P O}-\mathbf{B F}_{2} ; \mathbf{9 0} \%$ for NHPO-BF${ }_{2}$ ).

PHPO-BF $2 .{ }^{1} \mathrm{H}$ NMR $\left(400 \mathrm{MHz}, \mathrm{CDCl}_{3}\right) \delta 8.77(\mathrm{~d}, J=8.1 \mathrm{~Hz}$, $1 \mathrm{H}), 8.71(\mathrm{~d}, J=7.9 \mathrm{~Hz}, 1 \mathrm{H}), 8.61(\mathrm{~d}, J=8.3 \mathrm{~Hz}, 1 \mathrm{H}), 8.55(\mathrm{~d}, J=$ $7.9 \mathrm{~Hz}, 1 \mathrm{H}), 8.16(\mathrm{~d}, J=7.8 \mathrm{~Hz}, 2 \mathrm{H}), 7.95(\mathrm{t}, J=7.7 \mathrm{~Hz}, 1 \mathrm{H}), 7.79-$ $7.66(\mathrm{~m}, 3 \mathrm{H}), 7.66-7.54(\mathrm{~m}, 3 \mathrm{H}) .{ }^{13} \mathrm{C}$ NMR $\left(101 \mathrm{MHz}, \mathrm{CDCl}_{3}\right)$ $\delta$ 152.54, 145.16, 137.08, 134.06, 130.45, 129.44, 129.09, 128.92, 128.03, 127.86, 127.12, 126.62, 124.41, 123.23, 122.97, 122.74, 122.09. HRMS: calcd for $\mathrm{C}_{20} \mathrm{H}_{13} \mathrm{BF}_{2} \mathrm{~N}_{2} \mathrm{O}[\mathrm{M}+\mathrm{H}]^{+} 347.1176$, found 347.1165.

NHPO-BF $2 .{ }^{1} \mathrm{H}$ NMR $\left(400 \mathrm{MHz}, \mathrm{CDCl}_{3}\right) \delta 8.78(\mathrm{~m}, 2 \mathrm{H}), 8.72(\mathrm{~s}$, $1 \mathrm{H}), 8.62(\mathrm{~d}, J=8.3 \mathrm{~Hz}, 1 \mathrm{H}), 8.56(\mathrm{~d}, J=8.0 \mathrm{~Hz}, 1 \mathrm{H}), 8.27(\mathrm{dd}, J=$ 9.0, 2.0 Hz, 1H), 8.12-8.01 (m, 2H), 7.95 (m, 2H), 7.83-7.67 (m, $3 \mathrm{H}), 7.67-7.58(\mathrm{~m}, 2 \mathrm{H}) .{ }^{13} \mathrm{C}$ NMR $\left(101 \mathrm{MHz}, \mathrm{CDCl}_{3}\right) \delta 152.28$, 142.65, 137.10, 134.22, 134.09, 133.86, 133.12, 129.66, 129.60, 129.17, 129.01, 128.07, 127.93, 127.89, 127.31, 127.22, 126.75, 124.50, 123.32, 123.21, 123.07, 122.23, 119.36. HRMS: calcd for $\mathrm{C}_{24} \mathrm{H}_{15} \mathrm{BF}_{2} \mathrm{~N}_{2} \mathrm{O}[\mathrm{M}+\mathrm{H}]^{+}$397.1342, found 397.1323.

\section{Results and discussion}

The photophysical properties of $\mathbf{P H P O}-\mathbf{B F}_{2}$ and $\mathbf{N H P O}-\mathbf{B F}_{2}$ were studied in various organic solvents with different polarity. The absorption band of PHPO-BF ${ }_{2}$ was centred at $480 \mathrm{~nm}$ in THF, but shifts to $496 \mathrm{~nm}$ in chloroform (Fig. 1A). Its absorptions in hexane and acetonitrile were found at 490 and $491 \mathrm{~nm}$, respectively. For NHPO-BF 2 (Fig. 1C), the maximum absorption bands were found at 505, 514, 496, $510 \mathrm{~nm}$ in hexane, chloroform, THF and acetonitrile, respectively. It seems that the absorption of the two boron-difluoride dyes was non-dependent on the polarity of the solvent, indicating that intramolecular charge transfer (ICT) should be not significant for these two compounds. This speculation is accordance to the result of density functional theory (DFT) calculation (Fig. S1†). For both boron-difluoride dyes, the HOMO and LUMO distributions were donated by all atomic orbitals in the whole $\pi$ system, and there was only slight difference between the HOMO and LUMO orbitals.

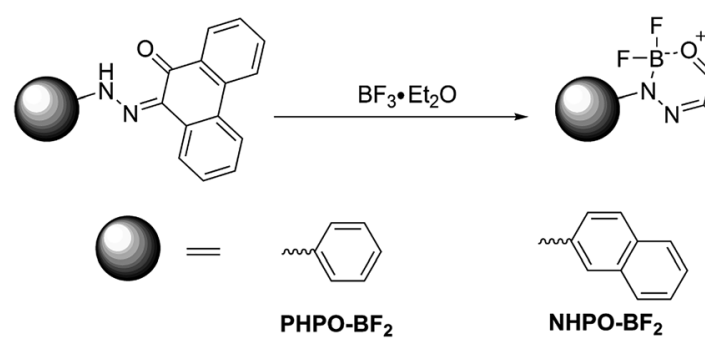

Scheme 1 Synthesis of PHPO- $\mathrm{BF}_{2}$ and $\mathrm{NHPO}-\mathrm{BF}_{2}$. 

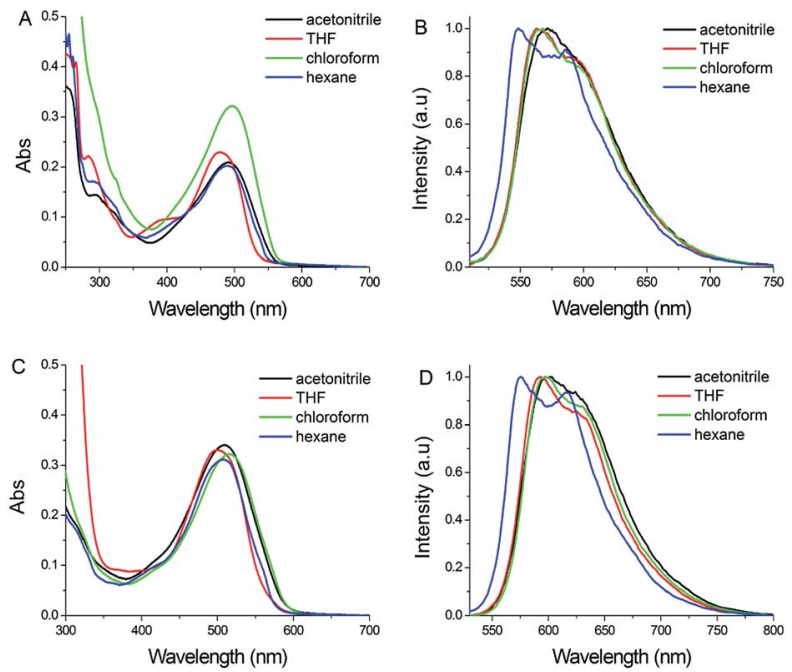

Fig. 1 (A) Absorption and (B) fluorescence spectra of PHPO-BF 2 in different solvents; (C) absorption and (D) fluorescence spectra of $\mathrm{NHPO}-\mathrm{BF}_{2}$ in different solvents $\left(1.0 \times 10^{-5} \mathrm{M}\right)$.

Fluorescence spectra of $\mathbf{P H P O}-\mathbf{B F}_{2}$ showed two emissive bands in hexane, centred at 546 and $586 \mathrm{~nm}$, respectively (Fig. 1B). Concentration-dependent fluorescence in hexane indicates that there was only one main emissive peak $(551 \mathrm{~nm})$ in highly dilute hexane solution (Fig. S2†). With concentration increasing, the emission around $580 \mathrm{~nm}$ appeared. Considering the planar structure of the phenanthrene unit in the borondifluoride dye, significant intermolecular $\pi-\pi$ interactions might be present to induce the fluorescence red-shift, and $\mathrm{J}$ or $\mathrm{H}$ aggregates might be formed in concentrated hexane solutions. Solvent polarity usually affects the fluorescence of $\mathrm{D}-\pi-\mathrm{A}$ dyes significantly, but no obvious fluorescence difference was found in solvents with different polarity, due to the inapparent ICT character of these two dyes (Fig. 1B and D).

Concentration-dependent absorption and fluorescence in THF was also measured to investigate the evolution of molecular assembly of these two dyes, which can provide more information about aggregation. The fluorescence of $\mathbf{P H P O}-\mathbf{B F}_{2}$ in dilute THF solution $\left(1.0 \times 10^{-7} \mathrm{M}\right)$ is centred at $613 \mathrm{~nm}$. When the concentration increases to $1.0 \times 10^{-5} \mathrm{M}$, the fluorescence blue-shifts to $566 \mathrm{~nm}$. If its concentration further increases, red-shift of the emission was observed (Fig. S3†). It seems that $\mathbf{P H P O}-\mathbf{B F}_{2}$ tends to form $\mathrm{H}$-aggregate first in concentrated THF solution $\left(C<5.0 \times 10^{-4} \mathrm{M}\right)$, which is also supported by concentration-dependent absorption spectra (Fig. S3A $\dagger$ ). In dilute solution, there appears broad absorption band ranging from 500-700 $\mathrm{nm}$, and this broad absorption band disappears in concentrated solution. But in highly concentrated THF solution $\left(C>5.0 \times 10^{-4} \mathrm{M}\right)$, different stacking modes might coexist to form both $\mathrm{H}$-aggregate and $\mathrm{J}$ aggregate, resulting in red-shift of the fluorescence.

For NHPO-BF 2 , it emits at $612 \mathrm{~nm}$ in dilute THF solution $(1.0 \times$ $10^{-7} \mathrm{M}$ ), and it blue-shifts to $590 \mathrm{~nm}$ when the concentration

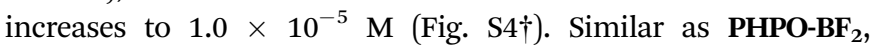
significant red-shift was observed with concentration further increasing. It seems that $\mathbf{N H P O}-\mathbf{B F}_{2}$ tends to form $\mathrm{H}$-aggregate when the concentration is between $1.0 \times 10^{-7} \mathrm{M}$ and $1.0 \times$ $10^{-4} \mathrm{M}$, and then J-aggregate in more concentrated solutions. The slight different molecular assembly behavior could be ascribed to the different $\pi$ system of phenyl unit and naphthyl group.

Most boron-difluoride compounds (such as BODIPY dyes) are highly fluorescent in dilute solutions, but prone to fluorescence quenching in the solid state due to intermolecular $\pi-\pi$ interactions. ${ }^{5}$ However, for the two boron-difluoride compounds, their fluorescences were very weak in dilute solutions (Table S1 $\dagger$ ), and their quantum yields were less than $0.1 \%$ in all the solvents studied (except in chloroform), possibly due to the non-irradiative decay via energy lost from the rotation of the aromatic rings (phenyl and naphthyl ring). In fact, their emissions in dilute solutions can hardly be observed by naked eyes. However, the solid powders of these two boron-difluoride compounds emit much stronger fluorescences and these emissions could be visible observed by a portable UV lamp. Since these two compounds emit stronger fluorescence in the solid state than in dilute solutions, we suppose they may exhibit AIEE characteristics.

The AIEE properties of two boron-difluorides were tested by absorption and fluorescence spectroscopy in a binary solvent system containing THF and water. Considering the hydrophobicity of boron-difluoride dyes, these luminogens should be able to precipitate in the aqueous mixtures with high water fractions $\left(f_{\mathrm{w}}\right)$, which was proved by the fact that these two compound exhibited Tyndall effect in mixed solutions containing large amount of water (Fig. S5 $\dagger$ ). As shown in Fig. 2B, as the water content $\left(f_{\mathrm{w}}\right)$ is from $0 \%$ to $10 \%$, the fluorescence intensity becomes weakened for $\mathbf{P H P O}-\mathbf{B F}_{2}$, possible due to the intermolecular interaction between water and the dye. With $f_{\mathrm{w}}$ between $10 \%$ and $70 \%$, the fluorescence intensity is gradually increased, and reached a maximum at $f_{\mathrm{w}} 80 \%\left(\Phi_{\mathrm{f}}=0.6 \%\right)$. With water amount further increasing, the fluorescence weakened again with a significant red shift. PHPO-BF $\mathbf{B}_{2}$ emits at $566 \mathrm{~nm}$ in dilute THF solution, but its fluorescence shifts to $660 \mathrm{~nm}$ when $f_{\mathrm{w}}$ reaches $99 \%\left(\Phi_{\mathrm{f}}=0.2 \%\right)$. It's clear that emissive aggregate of PHPO-BF ${ }_{2}$ has been formed at $f_{\mathrm{w}} 80 \%$, and it shows more intense fluorescence than in dilute THF solution due to the restriction of rotation to alleviate the radiationless relaxation channel.

To investigate the fluorescence quenching in $\mathrm{THF} / \mathrm{H}_{2} \mathrm{O}$ containing large amount of water, particle size measurement for these samples was performed (Fig. S6†). The size distribution of PHPO-BF $\mathbf{B F}_{2}$ in the solution containing $80 \%$ water is in a normal fashion with average size around $440 \mathrm{~nm}$. But for the sample containing $99 \%$ water, a much wider size distribution was observed, implying that it might agglomerate to form amorphous aggregate. The formation of amorphous aggregate should be responsible for the decreased fluorescence intensity. On the other hand, the red-shift of the fluorescence in $\mathrm{THF} / \mathrm{H}_{2} \mathrm{O}$ containing large amount of water might be aroused by the formation of J-aggregate. The absorption measurement also supports this hypothesis, because a shoulder band around $570 \mathrm{~nm}$ appeared (Fig. 2A).

For NHPO-BF $\mathbf{B}_{2}$, there is no significant fluorescence change with $f_{\mathrm{w}}$ between $0 \%$ to $60 \%$. However, the fluorescence intensity gets greatly enhanced when $f_{\mathrm{w}}$ is beyond $70 \%\left(\Phi_{\mathrm{f}}=1.3 \%\right)$, and 

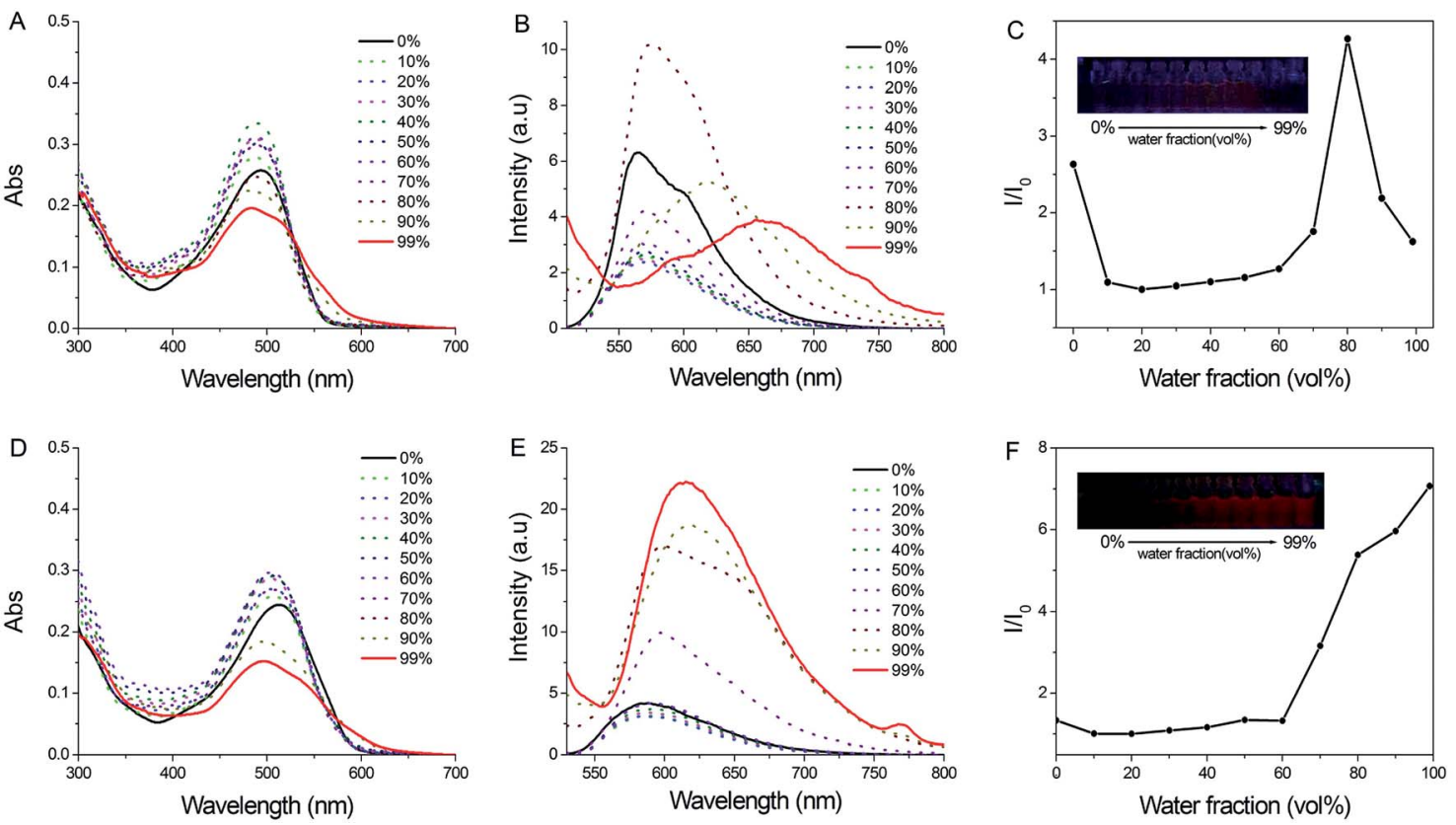

Fig. 2 (A) Absorption, (B) fluorescence and (C) relative fluorescent intensity $\left(/ / I_{0}\right)$ change of $\mathrm{PHPO}-\mathrm{BF} \mathrm{F}_{2}$ in $\mathrm{THF} / \mathrm{H}_{2} \mathrm{O}$ mixed solution with different water fraction; (D) absorption, (E) fluorescence and (F) relative fluorescent intensity $\left(/ / I_{0}\right)$ change of $\mathrm{NHPO}-\mathrm{BF}_{2}$ in $\mathrm{THF} / \mathrm{H}_{2} \mathrm{O}$ mixed solution with different water fraction $\left(1.0 \times 10^{-5} \mathrm{M}\right.$; inset: fluorescent images).

the fluorescence intensity always increases with water content increasing. Meanwhile, a slight red shift of fluorescence occurs, and a new shoulder absorption band appeared around $600 \mathrm{~nm}$, manifesting the formation of J-aggregate. Compared to PHPO$\mathbf{B F}_{2}$, no fluorescence quenching was observed during the aggregation even at $f_{\mathrm{w}} 99 \%\left(\Phi_{\mathrm{f}}=3.1 \%\right)$, possibly due to the formation of comparatively smaller particles with uniform size distribution (Fig. S7†).

In order to understand the AIEE characters of these two boron-difluoride dyes, single crystal of $\mathbf{P H P O}-\mathbf{B F}_{2}$ suitable for Xray single crystal diffraction was cultivated from a mixed solvent of dichloromethane and petroleum ether. As shown in Fig. 3, the $\mathrm{B}-\mathrm{N}$ bond distance is measured to be $1.59 \AA$, and the $\mathrm{B}-\mathrm{O}$ bond length is $1.46 \AA$, manifesting the asymmetry of the molecule. The F-B-F group acted as a bifurcated acceptor with an angle of $112.18^{\circ}$. As a fluorescent chromophore, the conjugated $\mathrm{BF}_{2}$ chromophore exhibits good planarity with the mean deviation from the whole $\pi$ system $0.057 \AA$ (except for the B-F moiety and the phenyl ring). The angle between the chromophore plane and $\mathrm{O}-\mathrm{B}-\mathrm{N}$ plane is estimated to be $31.21^{\circ}$, and the angle between the chromophore plane and the phenyl ring is $47.14^{\circ}$, demonstrating a non-planar molecular conformation (Fig. S8 $\dagger$ ). Due to the high planarity of the phenanthrene ring, intermolecular $\pi-\pi$ interaction between neighbouring phenanthrene rings is present in the crystal structure. In fact, a trimeric "sandwich"-like structure was found. The phenanthrene ring in one $\mathbf{P H P O}-\mathbf{B F}_{2}$ molecule overlaps with the phenanthrene rings in other two neighbouring molecules. It's noteworthy that the $\pi-\pi$ overlapping only exists between phenanthrene ring, possibly due to that the phenyl ring is not in the same plane with the chromophore plane. The intermolecular $\pi-\pi$ interactions in molecular clusters were estimated by density functional theory (DFT) calculation using M062x functional (shown as "green cloud" in Fig. 3C). Aided by the $\pi-\pi$ stacking between the phenanthrene ring in $\mathbf{P H P O}-\mathbf{B F}_{2}$ molecule, one-dimensional stacking mode was formed. But this one-dimensional stacking has two kinds of different aggregations. Two PHPO-BF 2 molecules in one packing type adopt an antiparallel arrangement with interplanar distance $3.38 \AA$, and most of the whole phenanthrene ring overlaps with another one, representing $\mathrm{H}$-aggregation. Another two molecules have a longer interplanar distance of $3.42 \AA$ with partial overlapping, indicating J-aggregation. ${ }^{47}$ Since the overlapping of the intermolecular $\pi$ system is partial, the crystal emits visible longwavelength aggregate emission with quantum yield $3.0 \%$ ( $\tau$ $3.34 \mathrm{~ns})$.
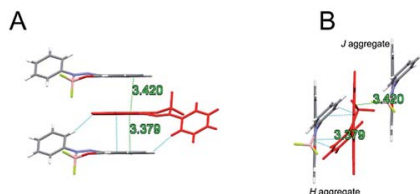

C

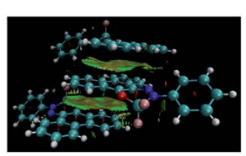

D
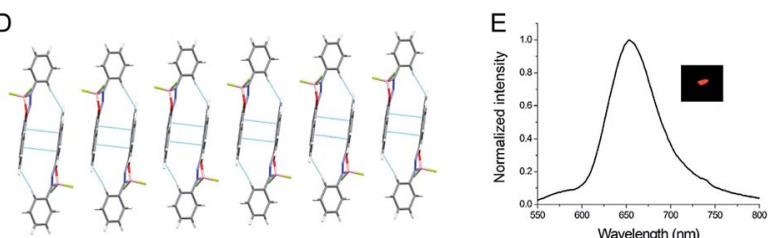

Fig. 3 Single crystal of PHPO-BF 2 (A) side view along $x$ axial; (B) side view along $z$ axial; (C) intermolecular interactions (shown as green cloud); (D) one-dimensional stacking; (E) fluorescent spectra and image. 

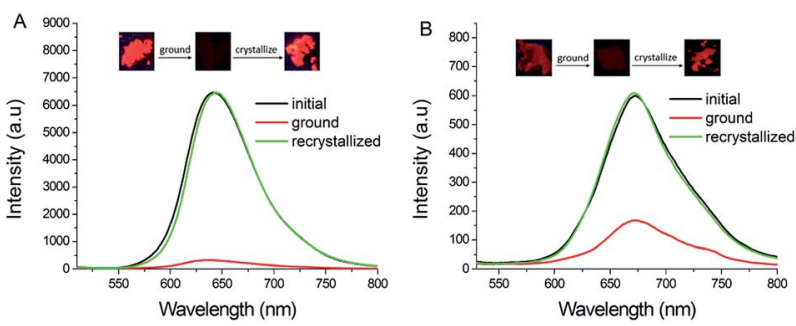

Fig. 4 (A) Fluorescent spectra and images of $\mathrm{PHPO}-\mathrm{BF}_{2}$ during mechanochromism; (B) fluorescent spectra and images of $\mathrm{NHPO}-\mathrm{BF}_{2}$ during mechanochromism.

Considering that PHPO-BF 2 could form emissive and nonemissive aggregates during the precipitating process in THF, we suppose these boron-difluoride derivatives may be able to exhibit reversible mechanofluorochromism. As shown in Fig. 4, the initial powder of PHPO-BF $\mathbf{F}_{2}$ emits visible red fluorescence at $642 \mathrm{~nm}$ with lifetime $3.46 \mathrm{~ns}$. The fluorescence quantum yield is estimated to be $4.6 \%$. Upon mechanical grinding in a mortar, the fluorescence intensity gradually weakens and turns almost completely quenched and invisible by naked eyes. Crystallization from mixture of dichloromethane and petroleum ether or fume by dichloromethane or heat could recover its fluorescence, manifesting reversible mechanochromism. Similar

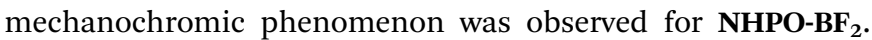
The initial powder of $\mathbf{N H P O}-\mathbf{B F}_{2}$ emits intense near-infrared fluorescence at $672 \mathrm{~nm}\left(\Phi_{\mathrm{f}}: 1.5 \%, \tau: 1.30 \mathrm{~ns}\right)$. The emission of the initial powder was quenched significantly after grinding, and it could be recovered completely by recrystallization.

In order to investigate the mechanochromic mechanism of these two boron-difluoride dyes, all samples were subjected to XRD measurements. The initial solid powder of PHPO-BF exhibits intense and sharp reflections, demonstrating a crystalline structure (Fig. 5). Notably, the reflections of the initial powder resemble most peaks of the simulated peaks of the single crystal (Fig. S9†), revealing that the initial powder might possess similar molecular packing mode with that in single crystal. After grinding in the mortar, most diffraction peaks disappeared or significantly weakens, suggesting that the ground powder turns amorphous. Currently, it's difficult to understand the molecular packing modes that determined amorphous samples' fluorescence properties. Considering that the structure of PHPO-BF $\mathbf{F}_{2}$ optimized by B3LYP with the 6-
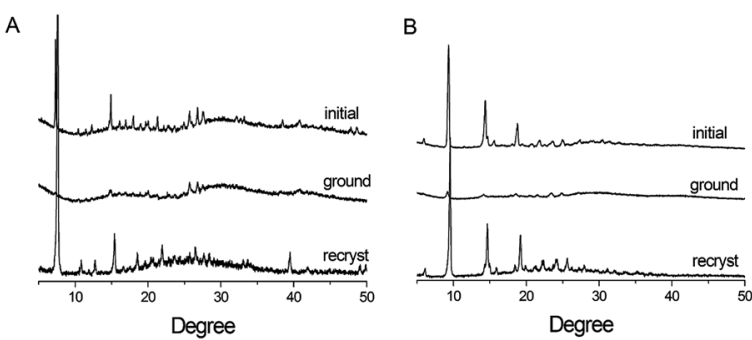

Fig. 5 XRD patterns of $\mathrm{PHPO}^{-\mathrm{BF}_{2}}$ (A) and $\mathrm{NHPO}^{-\mathrm{BF}_{2}}$ (B) during mechanochromism.

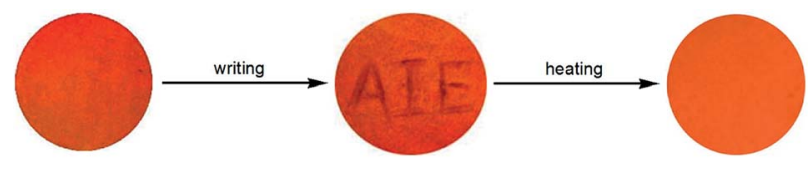

Fig. 6 Information writing and erasing processes.

$31+\mathrm{G}(\mathrm{d}, \mathrm{p})$ basis sets exhibits better planarity compared to that in single crystal, we suppose the planar structure of $\mathbf{P H P O}-\mathbf{B F}_{2}$ might facilitate the formation of J-aggregate with more intense intermolecular $\pi-\pi$ interactions (shorter interplanar distance and greater overlapping degree), which could quench the fluorescence. The hypothesis is further supported by the potential molecular packing obtained by polymorph prediction, and the intermolecular $\pi-\pi$ interactions turn much more intense for the conformation with planar structure (Fig. S10†). Thus, we propose that the grinding might intensify the intermolecular $\pi$ $\pi$ interactions, and the mechanochromic mechanism might be the reversible conversion from emissive $\mathrm{H}(\mathrm{J})$-aggregate with medium intermolecular $\pi-\pi$ stacking to non-emissive $\mathrm{J}$ aggregate with intense intermolecular $\pi-\pi$ interactions. Similar mechanochromic phenomenon is observed for NHPO$\mathbf{B F}_{2}$. Therefore, the fluorescence changes of $\mathbf{P H P O}-\mathbf{B F}_{2}$ and $\mathbf{N H P O}_{\mathbf{B}} \mathbf{B F}_{2}$ upon mechanical force could be ascribed to the stimuli-induced changes of intermolecular $\pi-\pi$ interactions.

Since these two AIEE boron-difluoride dyes showed highcontrast mechanochromic properties, they might be adopted for applications in memory field. Here, $\mathbf{P H P O}-\mathbf{B F}_{2}$ is chosen as an example to demonstrate its application in optical recording. First, a filter paper was immersed in a concentrated solution of PHPO-BF ${ }_{2}$ in chloroform, and then dried. The initial paper emits intense red fluorescence. A black "AIE" stamp could be written on the red background using a stainless steel spatula (Fig. 6). The stamp could be easily erased upon fuming with dichloromethane or heating. And the recovered paper could be used for imputing information again.

\section{Conclusions}

We synthesized two novel boron-fluoride derivatives, using phenanthrenequinone hydrazone derivatives as ligands. These two dyes emit weak fluorescence in dilute solutions, but enhanced emissions were observed in aggregate state. Owing to the asymmetric structure, these boron-difluorides possess comparatively large stokes shift (over $70 \mathrm{~nm}$ ). Because the phenyl (naphthyl) ring could rotate to provide planar or nonplanar conformations, multiple stacking modes with different intermolecular $\pi-\pi$ interactions were found in their aggregate state. The conformation in single crystal structure of $\mathbf{P H P O}-\mathbf{B F}_{2}$ is non-planar and the intermolecular $\pi-\pi$ interaction is medium, making the crystal emit visible red fluorescence. In a planar conformation, intense intermolecular $\pi-\pi$ interactions exists to quench the fluorescence, which is supported by polymorph prediction. Grinding might change the conformation of these molecules to intensify the intermolecular interactions, and then quench their fluorescences. Therefore, the 
mechanochromic mechanism of these boron-difluoride dyes is ascribed to the stacking model transition between $\mathrm{H}(\mathrm{J})$ aggregation formation with loose molecular packing and Jaggregation with intense intermolecular interactions.

\section{Conflicts of interest}

There are no conflicts to declare.

\section{Acknowledgements}

We are grateful for the financial support from the National Natural Science Foundation of China (21472111) for financial support. The authors thank Dr Y. Lu from East China University of Science and Technology for the quantum calculation.

\section{Notes and references}

1 M. Mao, S. Xiao and J. Li., Tetrahedron, 2012, 68, 5037-5041. 2 Y. Deng, Y. Y. Cheng, H. Liu, J. Mack, H. Lu and L. G. Zhu, Tetrahedron Lett., 2014, 55, 3792-3796.

3 J. F. Araneda, W. E. Piers, B. Heyne, M. Parvez and R. McDonald, Angew. Chem., Int. Ed., 2011, 50, 12214-12217.

4 H. Liu, H. Lu, J. Xu, Z. P. Liu, Z. F. Li, J. Mack and Z. Shen, Chem. Commun., 2014, 50, 1074-1076.

5 S. Z. Xiao, Q. Cao and F. J. Dan, Curr. Org. Chem., 2012, 16, 2970-2981.

6 S. Wang, R. H. Tan, Y. Q. Li, Q. Li and S. Z Xiao, Dyes Pigm., 2016, 132, 342-346.

7 S. Wang, H. C Lan, S. Z. Xiao, R. H. Tan and Y. X. Lu, Chem.Asian J., 2017, 12, 198-202.

8 S. Wang, S. Z Xiao, X. H. Chen, R. H. Zhang, Q. Cao and K. Zou, Dyes Pigm., 2013, 99, 543-547.

9 X. H. Chen, S. Z. Xiao, S. Wang, Q. Cao, K. Zou, N. Y. Huang and Z. S. Deng, Tetrahedron Lett., 2013, 54, 4116-4120.

10 Q. Cao, S. Z. Xiao, M. F. Mao, X. H. Chen, S. Wang, L. Li and K. Zou, J. Organomet. Chem., 2012, 717, 147-151.

11 M. F. Mao, S. Z. Xiao, J. F. Li, Y. Zou, R. H. Zhang, J. R. Pan, F. J. Dan, K. Zou and T. Yi, Tetrahedron, 2012, 68, 5037-5041.

12 H. Liu, H. Lu, Z. K. Zhou, S. Shimizu, Z. F. Li, N. Kobayashi and Z. Shen, Chem. Commun., 2015, 51, 1713-1716.

13 J. Luo, Z. Xie, J. W. Y. Lam, L. Cheng, H. Chen, C. Qiu, H. S. Kwok, X. Zhan, Y. Liu, D. Zhu and B. Z. Tang, Chem. Commun., 2001, 18, 1740-1741.

14 Y. P. Qi, Y. T. Wang, G. X. Ge, Z. Y. Liu, Y. J. Yu and M. Xue, J. Mater. Chem. C, 2017, 5, 11030-11038.

15 J. B. Su, W. P. Chen, F. Wu, X. Y. Bai, W. W. Du, L. P. Fang, Y. G. Wu, X. Wen, H. J. Wang and X. W. Ba, Dyes Pigm., 2019, 162, 808-814.

16 H. Z. Gao, D. F. Xu, Y. H. Wang, X. L. Liu, A. X. Han and C. Zhang, Dyes Pigm., 2018, 150, 59-66.

17 L. Zhai, F. S. Zhang, J. B. Sun, M. Y. Liu, M. Sun and R. Lu, Dyes Pigm., 2017, 145, 54-62.

18 K. Suenaga, K. Tanaka and Y. Chujo, Eur. J. Org. Chem., 2017, 35, 5191-5196.

19 Z. Q. Zhang, Z. Wu, J. B. Sun, P. C. Xue and R. Lu, RSC Adv., 2016, 6, 43755-43766.
20 D. Wu, L. Shao, Y. Li, Q. L. Hu, F. H. Huang, G. C. Yu and G. P. Tang, Chem. Commun., 2016, 52, 541-544.

21 Q. S. Liu, X. Q. Wang, H. Yan, Y. P. Wu, Z. Y. Li, S. W. Gong, P. Liu and Z. P. Liu, J. Mater. Chem. C, 2015, 3, 2953-2959.

22 K. Perumal, J. A. Garg, O. Blacque, R. Saiganesh, S. Kabilan, K. Kuppusamy and K. Venkatesan, Chem.-Asian J., 2012, 7, 2670-2677.

23 Y. Meesala, V. Kavala, H. C. Chang, T. S. Kuo, C. F. Yao and W. Z. Lee, Dalton Trans., 2015, 44, 1120-1129.

24 S. W. Gong, Q. S. Liu, X. Q. Wang, B. Xia, Z. P. Liu and W. J. He, Dalton Trans., 2015, 44, 14063-14070.

25 K. I. Lugovik, A. K. Eltyshev, P. O. Suntsova, P. A. Slepukhin, E. Benassi and N. P. Belskaya, Chem.-Asian J., 2018, 2, 311324.

26 X. Q. Wang, Y. P. Wu, Q. S. Liu, Z. Y. Li, H. Yan, C. L. Ji, J. C. Duan and Z. P. Liu, Chem. Commun., 2015, 51, 784-787.

27 R. H. Tan, Q. F. Lin, Y. Wen, S. Z. Xiao, S. Wang, R. H. Zhang and T. Yi, CrystEngComm, 2015, 17, 6674-6680.

28 R. R. Maar and J. B. Gilroy, J. Mater. Chem. C, 2016, 4, 64786482.

29 X. L. Zhu, R. Liu, Y. H. Li, H. Huang, Q. Wang, D. F. Wang, X. Zhu, S. S. Liu and H. J. Zhu, Chem. Commun., 2014, 50, 12951-12954.

30 J. Zheng, F. Huang, Y. J. Li, T. W. Xu, H. Xu, J. H. Jia, Q. Ye and J. R. Gao, Dyes Pigm., 2015, 113, 502-509.

31 L. Quan, Y. Chen, X. J. Lv and W. F. Fu, Chem.-Eur. J., 2012, 18, 14599-14604.

32 Y. Yang, X. Su, C. N. Carroll and I. Aprahamian, Chem. Sci., 2012, 3, 610-613.

33 Y. P. Wu, Z. Y. Li, Q. S. Liu, X. Q. Wang, H. Yan, S. W. Gong, Z. P. Liu and W. J. He, Org. Biomol. Chem., 2015, 13, 57755782.

34 Y. Kubota, S. Tanaka, K. Funabiki and M. Matsui, Org. Lett., 2012, 14, 4682-4685.

35 R. Yoshii, A. Hirose, K. Tanaka and Y. Chujo, Chem.-Eur. J., 2014, 20, 8320-8324.

36 P. Choudhury, S. Sarkar and P. K. Das, Langmuir, 2018, 34, 14328-14341.

37 A. S. V. Anand, K. Mahesh and V. Priyanka, J. Mater. Sci.: Mater. Electron., 2018, 29, 10949-10961.

38 Y. Zhang, J. H. Wang, W. Zheng, T. Chen, Q. X. Tong and D. Li, J. Mater. Chem. A, 2014, 2, 4159-4166.

39 B. K. An, S. K. Kwon, S. D. Jung and S. Y. Park, J. Am. Chem. Soc., 2002, 124, 14410-14415.

40 Q. Zhao, L. Li, F. Y. Li, M. X. Yu, Z. P. Liu, T. Yi and C. H. Huang, Chem. Commun., 2008, 6, 685-687.

41 Y. Deng, W. Yuan and Z. Jia, J. Phys. Chem. B, 2014, 118, 14536-14545.

42 Gaussian 09, Revision D.01, Gaussian, Inc., Wallingford CT, 2013.

43 Materials Studio, Accelrys Software Inc., San Diego, 2013.

44 Y. Zhao and D. G. Truhlar, Acc. Chem. Res., 2008, 41, 157-167. 45 T. Lu and F. W. Chen, J. Comput. Chem., 2012, 33, 580-592. 46 X. J. Yan, P. C. Zhu, Y. Li, S. Yuan, H. C. Lan and S. Z. Xiao, Mater. Today Commun., 2019, 20, 100565.

47 Y. B. Shen, P. Chen, J. X. Liu, J. P. Ding and P. C. Xue, Dyes Pigm., 2018, 150, 354-362. 